\title{
Effect of E-Beams Irradiation Dose on the Sensing Properties of Pt-Functionalized Reduced Graphene Oxides Following Annealing of the 6 nm-Thick Pt Layer
}

\author{
Yong Jung Kwon ${ }^{1}$, Ali Mirzaei ${ }^{2}$, Han Gil Na ${ }^{1}$, Sung Yong Kang ${ }^{1}$, Myung Sik Choi ${ }^{1}$, Jae Hoon Bang ${ }^{1}$, \\ Jaegab Lee ${ }^{3}$, Inpil Kang ${ }^{4}$, Sang Sub Kim ${ }^{5, *}$, and Hyoun Woo Kim ${ }^{1,2, *}$ \\ ${ }^{1}$ Division of Materials Science and Engineering, Hanyang University, Seoul 04763, Republic of Korea \\ ${ }^{2}$ The Research Institute of Industrial Science, Hanyang University, Seoul 04763, Republic of Korea \\ ${ }^{3}$ School of Advanced Materials Engineering, Kookmin University, Seoul 02707, Republic of Korea \\ ${ }^{4}$ Department of Mechanical Design Engineering, Pukyong National University, Busan 48547, Republic of Korea \\ ${ }^{5}$ Department of Materials Science and Engineering, Inha University, Incheon 22212, Republic of Korea
}

\begin{abstract}
Pt-functionalized RGO samples were prepared and subsequently irradiated with 2-MeV electron beams (e-beams). The effect of e-beam dose on the structural, electrical, and gas sensing properties of the samples was investigated. E-beam irradiation was found to induce significant microstructural changes in the Pt-functionalized RGO. Measurements of $\mathrm{NO}_{2}$ sensing at room temperature showed that the dose of 100 $\mathrm{kGy}$ led to better $\mathrm{NO}_{2}$ sensing properties compared to those of unirradiated sensors. On the other hand, the dose of $500 \mathrm{kGy}$ appeared to be excessive, leading to extensive damage of the RGO structure and a significant reduction in the response to $\mathrm{NO}_{2}$ gas. E-beam irradiation can be beneficial for improving the gas sensing of Pt-functionalized RGO, but its dose needs to be optimized to obtain the best sensing properties.
\end{abstract}

(Received November 1, 2016; Accepted December 19, 2016)

Keywords: reduced graphene oxide, gas sensor, E-Beam irradiation, Pt nanoparticles, $\mathrm{NO}_{2}$.

\section{INTRODUCTION}

The nano era has been, a tremendous increase in the number of reports discussing methods of fabricating and characterizing a variety of nanomaterials and nanostructures [1-20]. Among these, a new generation of gas sensors based on graphene materials such as graphene oxide, and reduced graphene oxide (RGO) have drawn attention, due to their room temperature operation, facile synthesis, and the ease with which their electrical properties can be tailored [21]. RGO-based gas sensors based on noble metal nanoparticles (NPs), used as either composite materials or decorating particles, have demonstrated better sensing properties than pristine RGO sensors [22,23]. This performance enhancement is due to the increase in electronic and chemical sensitivity provided by the noble metals. The electronic sensitization originates from the flow of electrons between the sensing

* Corresponding Author: Sang Sub Kim, Hyoun Woo Kim [Tel: +82-32-960-7546, E-mail: Sangsub@inha.ac.kr] [Tel: +82-2-2220-0382, E-mail: hyounwoo@hanyang.ac.kr] Copyright (c) The Korean Institute of Metals and Materials material and the metal NPs. The chemical sensitization is due to the catalytic effect of the metal NPs; the metal NPs can facilitates either the adsorption of target gases or their efficient transport to the sensing material. Furthermore, they induce increased interactions with preadsorbed oxygen species, facilitating the relevant chemical reactions [24]. However, for advanced applications, the sensing properties of RGO-based chemiresistive sensors still need further enhancement.

To enhance the sensing properties of gas sensors, a number of post-treatment modifications have been explored, including exposure to highly energetic radiation such as e-beams [25], ion beams [26], and gamma rays [27]. Different types of irradiation can produce significant microstructural and morphological changes in various materials such as polymers, ceramics, and semiconductors. More specifically, they can create a wide variety of defects in the materials, which ultimately modify their optical, electrical, and sensing properties [28]. For example, gamma radiation has been observed to decrease the electron mobility of $n-G a N$ by 


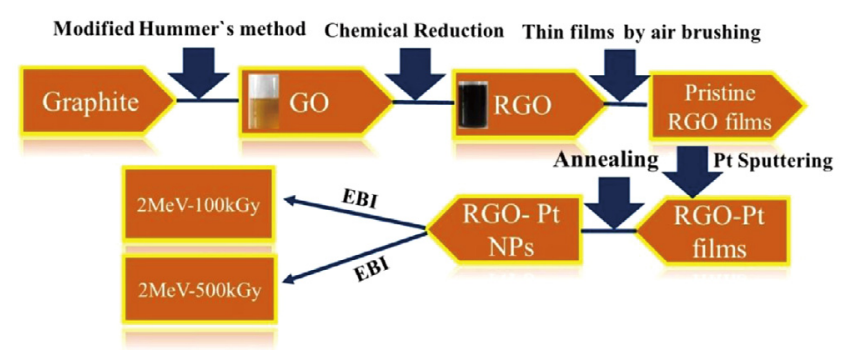

Fig. 1. Schematic illustration of synthesis processes.

introducing defects that act as scattering centers [29]. Yin et al. reported that indium vanadate nanoribbons irradiated with gamma rays showed enhanced ammonia sensing, which was attributed to the creation of defects [30]. Bal et al. reported that ion bombardment of a $\mathrm{ZnO}$ nanowire-based sensor decreased its response to ammonia gas and increased its recovery speed. They attributed these results to the rupturing of the nanostructure under irradiation, which decreased porosity and hindered the diffusion of gas molecules into the inner part of the sample [31]. Kwon et al. attributed improvement in the gas sensing properties of pristine RGO after e-beam irradiation to the formation of defects [32].

Among the various irradiation methods, e-beams have recently been extensively studied as a means of preparing noble metal NPs [33], bimetallic NPs [34], and core-shell NPs [35], and have also been used to controllably introduce defects in order to modify materials' optical and electrical properties [36].

E-beams lose their energy when passing through materials due to interactions with atoms. At lower energies, elastic collisions of e-beams and atoms are the dominant form of loss, whereas at higher energies inelastic collisions between the energetic ions and atoms are dominant [26]. High-dose irradiation of materials leads to the formation of lattice defects such as vacancies, defect clusters, and dislocations. These defects can act as recombination centers by trapping generated carriers. In addition, exposure to high levels of radiation leads to significant changes in the electrical characteristics of metal oxides. In particular, there are two important effects of e-beam radiation: these are transient effects due to electron-hole pair generation and permanent effects which change the crystal lattice of the material [37].
In the present study we used e-beams with $2 \mathrm{MeV}$ energy, applied at two different doses, 100 and $500 \mathrm{kGy}$. This level of high-energy e-beams can ionize RGO. Upon ionization, the generated ions capture negatively charged electrons or positively charged holes, creating defects. These defects cause changes in the microstructure and in the distribution of oxygen vacancies in the material. Radiation can also lead to the segregation and diffusion of atoms, leading to phase segregation and voids. Accordingly, e-beam radiation can be used to modify the physical and chemical properties of the irradiated material [37].

Although there have been numerous reports regarding the gas sensing properties of noble metal/RGO materials [38-40], to the best knowledge of the authors, there have been very few reports regarding the effect of e-beam irradiation on the sensing behavior of RGO-based sensors. Accordingly, the present work studies the effect of e-beam dose on the $\mathrm{NO}_{2}$ sensing behavior of Pt-functionalized RGO sensors. The RGO used was prepared by the chemical reduction of GO, and sensors were prepared by sputtering $6 \mathrm{~nm}$ thick Pt layers onto RGO, followed by thermal annealing. The effect of e-beam dose upon the structure of the RGO was investigated. Also, the $\mathrm{NO}_{2}$ sensing properties of various sensors were studied and compared, including sensors based on either pristine RGO or Pt-functionalized RGO, and between RGO those subjected to no irradiation or to irradiation at various doses.

\section{EXPERIMENTAL PROCEDURES}

\subsection{SYNTHESIS AND E-BEAM IRRADIATION}

Figure 1 schematically illustrates the RGO synthesis procedure. Hummers' method was employed to convert graphite into GO [41]; more details are given in our previous paper [42]. The graphite oxidation process was started by stirring graphite $(1 \mathrm{~g})$ in an oxidizing mixture of concentrated $\mathrm{H}_{2} \mathrm{SO}_{4}(46 \mathrm{ml})$ containing $\mathrm{KMnO}_{4}(12 \mathrm{~g})$ and $\mathrm{H}_{3} \mathrm{PO}_{4}(12 \mathrm{~g})$. This procedure increases the interlayer distance between the packed graphite layers, thereby facilitating exfoliation and separation of graphene sheets. A very fine brown powder was obtained after repeated washing and filtration; this was dried 

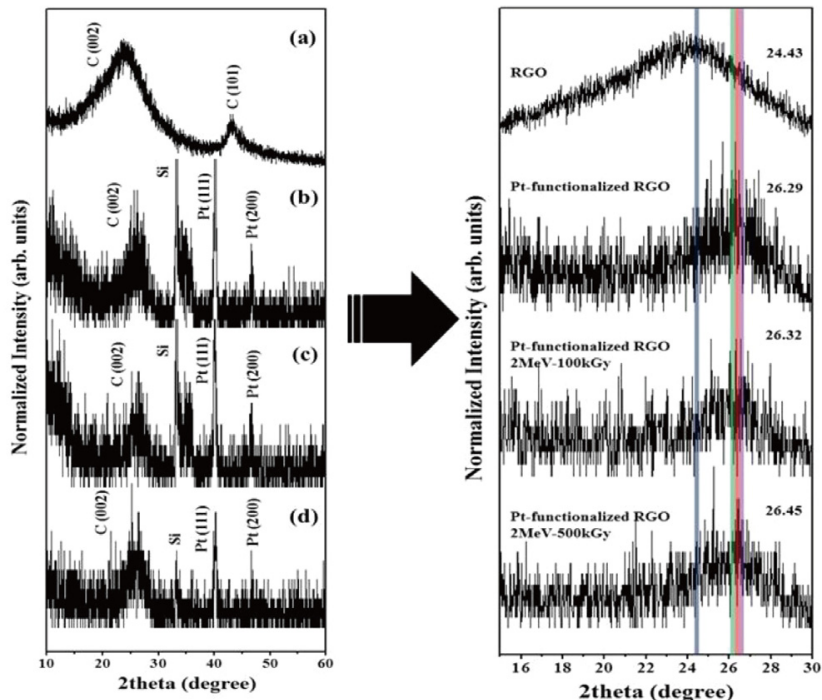

Fig. 2. (Left) XRD patterns of (a) pristine RGO, (b) unirradiated Pt-functionalized RGO, (c) Pt-functionalized RGO irradiated at the dose of $100 \mathrm{kGy}$, and (d) Pt-functionalized RGO irradiated at $500 \mathrm{kGy}$. (Right) Corresponding (002) peaks in the XRD patterns.

in a vacuum oven. The powder was mixed with hydrazine monohydrate and heated at $150{ }^{\circ} \mathrm{C}$ in an oil bath, yielding RGO. To prepare a homogenous suspension of RGO, it was dispersed in dimethylformamide $\left(\mathrm{C}_{3} \mathrm{H}_{7} \mathrm{NO}\right)$ and subsequently sonicated. The RGO suspension was coated onto quartz substrates, and then heated at $250{ }^{\circ} \mathrm{C}$ for $3 \mathrm{~h}$ to dry it. Annealing of the resulting RGO films was performed at 1100 ${ }^{\circ} \mathrm{C}$ for 30 min under a $100 \mathrm{sccm}$ flow of mixed $\mathrm{Ar}$ and $\mathrm{H}_{2}$ gas.

A turbo sputter coater with a circular Pt target (Emitech K575X, Emitech Ltd., Ashford, Kent, UK) was used to perform room-temperature coating of the Pt. To prepare $\mathrm{Pt}$ layers of $6 \mathrm{~nm}$ thickness on the RGO samples, plasma sputtering was conducted in the presence of Ar gas, using the controlled deposition time of $10 \mathrm{~s}$ and the DC sputter current of $65 \mathrm{~mA}$. Finally, to convert each Pt layer into a layer of Pt with NPs dispersed over the surface of the RGO, it was annealed at $700{ }^{\circ} \mathrm{C}$ for $0.5 \mathrm{~h}$ under a $2000 \mathrm{sccm}$ flow of $\mathrm{Ar}$ gas.

Irradiation of the Pt-functionalized RGO films was performed in air atmosphere at room temperature. E-beam irradiation was conducted at the ELV-8 electron accelerator (EBTech, Daejeon, Korea). The accelerating energy of 2 $\mathrm{MeV}$ was used with the two different doses of 100 and 500
kGy to study the effect of dose on the resulting films' physical properties and sensing behavior. The beam current was set at $1 \mathrm{~mA}$ and the pulse duration was fixed at $400 \mathrm{ps}$.

\subsection{MATERIAL CHARACTERIZATION}

To study the structure and phase formation of the prepared samples, X-ray diffraction (XRD) patterns were acquired under $\mathrm{Cu} \mathrm{K} \alpha(\lambda=1.541 \AA)$ radiation (Rigaku, Japan). To examine the morphological features of the Pt-functionalized RGO samples, we conducted scanning electron microscopy (SEM; JSM 5900 LV, JEOL, Japan), as well as transmission electron microscopy (HR-TEM; TECNAI 20, FEI, USA; with an accelerating voltage of $200 \mathrm{kV}$ ) coupled with energy dispersive spectrometry (EDS). Raman scattering under an Ar-ion laser source (532 nm, $2.33 \mathrm{eV}$ ) was measured in air at RT using a Horiba Jobin-Yvon LabRam HR Evolution system, equipped with a confocal microscope (100× microscope objective lens). A low laser power density of 265 $\mu \mathrm{W}$ at the sample surface was used to prevent laser-induced annealing effects. To analyze elemental compositions, X-ray photoelectron spectroscopy (XPS; VG Multilab ESCA 2000 system, UK) was conducted using a monochromatized $\mathrm{Al} \mathrm{K \alpha}$ $\mathrm{X}$-ray source $(\mathrm{h} v=1486.6 \mathrm{eV})$ at the Korean Basic Science Institute. The binding energies obtained from the XPS analysis were corrected for specimen charging by calibrating the $\mathrm{C} 1 \mathrm{~s}$ line to $284.5 \mathrm{eV}$.

\subsection{GAS SENSING MEASUREMENTS}

To enable gas sensing studies, double-layer electrodes of $\mathrm{Ni}$ ( $\sim 200 \mathrm{~nm}$ thickness) and $\mathrm{Au}$ ( $\sim 50 \mathrm{~nm}$ thickness) were sputtered onto sample films using an interdigitated electrode mask. The sensing setup used was similar to that previously described by our research group [43]. To test each sample's sensing properties, it was placed in a tubular furnace that was electrically connected to a measuring system (Keithley 2400) interfaced with a computer. The $\mathrm{NO}_{2}$ concentration (10 ppm) was precisely controlled by changing the mixing ratio of the $\mathrm{NO}_{2}$ and dry air using mass flow controllers, maintaining the total flow rate of $100 \mathrm{sccm}$. Resistances were measured in the presence of air $\left(\mathrm{R}_{\mathrm{a}}\right)$ and $\mathrm{NO}_{2}\left(\mathrm{R}_{\mathrm{g}}\right)$ at room temperature; the sensor response was defined as $R=R_{a} / R_{g}$. The response time 
was defined as the time required for the change in electrical resistance to reach $90 \%$ of the equilibrium value after the injection of $\mathrm{NO}_{2}$ gas, and the recovery time was defined as the time needed for the sensor to return to $90 \%$ of its initial resistance in air after removing the supply of $\mathrm{NO}_{2}$.

\section{RESULTS AND DISCUSSION}

\subsection{STRUCTURAL, MORPHOLOGICAL, AND CHEMICAL ANALYSES}

Figures $2 \mathrm{a}-\mathrm{d}$ show XRD patterns of the various sensors. Figure 2a shows an XRD spectrum of pristine RGO, in which only two carbon peaks were observed, namely the (002) and (101) peaks. On the other hand, one additional peak was observed in each of the spectra of the Pt-functionalized RGO samples (Figs. $2 \mathrm{~b}-\mathrm{d}$ ), ignoring the Si peaks originating from the substrate, which belonged to the $\{111\}$ plane of Pt. This peak confirms the formation of crystalline Pt in the samples. Magnified XRD spectra focusing on the (002) carbon peak are shown in the left part of Fig. 2. For the pristine RGO samples, samples of Pt-functionalized RGO with no irradiation, Pt-functionalized RGO irradiated at the dose of $100 \mathrm{kGy}$, and Pt-functionalized RGO irradiated at $500 \mathrm{kGy}$, the peak positions of the (002) plane were respectively located at $24.43^{\circ}, 26.29^{\circ}, 26.32^{\circ}$, and $26.45^{\circ}$. This means the peak positions were shifted to higher Bragg diffraction angles with increasing dose, which corresponds to a slight decrease in the lattice spacing. Possible reasons for this small decrease in lattice spacing include the presence of residual compression strains caused by either the sputtering process or the electron. E-beam irradiation can cause lattice strain because it can introduce of oxygen vacancies and surface defects [44].

SEM micrographs of each Pt-functionalized RGO sample clearly showed fine and dispersed Pt NPs in a matrix of carbon (Fig. 3). The unirradiated sample had a relatively smooth surface while the sample dosed at $100 \mathrm{kGy}$ had a slightly rougher surface. The sample that received the high e-beam dose of $500 \mathrm{kGy}$ showed a rougher and more complicated surface. However, the SEM images did not allow a more detailed evaluation of the effects of the e-beam
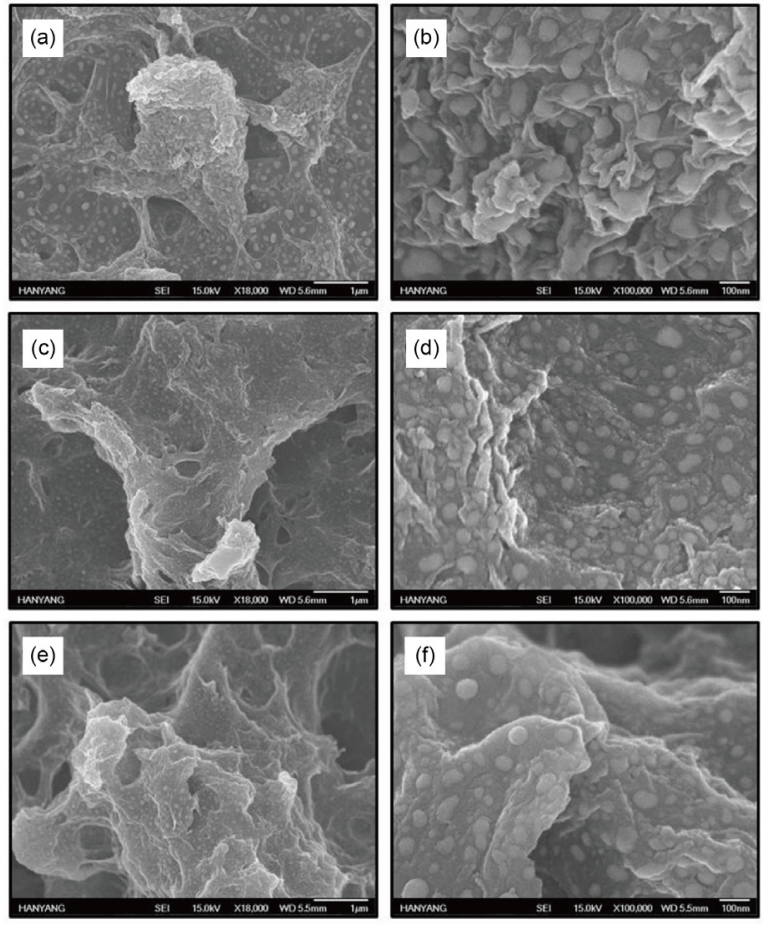

Fig. 3. Low- and high-magnification SEM images of (a, b) unirradiated Pt-functionalized RGO, (c, d) Pt-functionalized RGO irradiated at the dose of $100 \mathrm{kGy}$, and (e, f) Pt-functionalized RGO irradiated at $500 \mathrm{kGy}$.

irradiation on the final morphology. For that, further analysis using TEM was conducted.

In the TEM image of Pt-functionalized RGO irradiated at 100 kGy in Fig. 4a, RGO sheets functionalized with Pt NPs (5 $-50 \mathrm{~nm}$ ) can be easily seen. A HRTEM image showed that the spacing between the two lattice fringes was about 0.226 $\mathrm{nm}$ (Fig. 4b), which can be attributed to the (111) plane of Pt having a fcc crystal structure. In a selected area electron diffraction (SAED) pattern of this sample, ring patterns of the (101) and (002) planes of RGO and the (111), (200), and (220) planes of Pt confirmed the presence of both Pt and RGO in the sample (Fig. 4c). Elemental mapping analysis further verified the presence of fine and dispersed Pt NPs in a matrix of carbon (Figs. 4d-f). Similarly, in low-magnification TEM and HRTEM images of the sample irradiated with 500 kGy the existence of Pt NPs in a carbon matrix was evident (Fig. 5a, b). A lattice-resolved image of this sample shows multiple defects generated by the high-energy e-beams (Fig. $5 \mathrm{c}$; see red circles). In fact, if the kinetic energy transferred to 

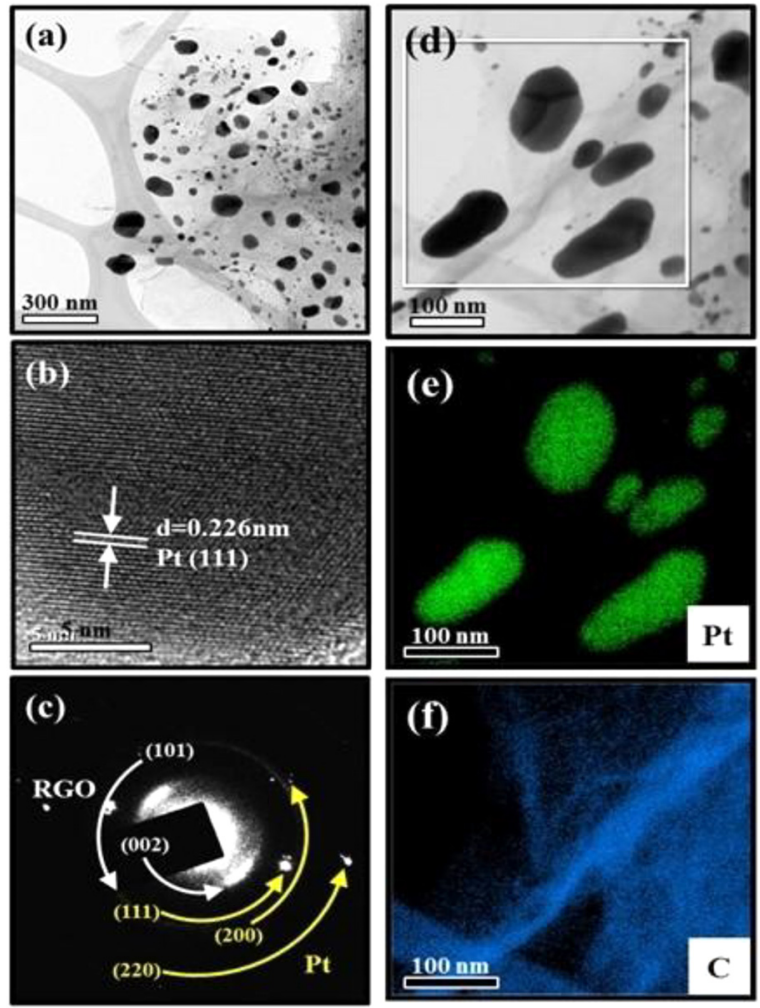

Fig. 4. (a) TEM image of Pt-functionalized RGO irradiated at the dose of $100 \mathrm{kGy}$. (b) Lattice-resolved-TEM image. (c) SAED pattern. (d) TEM image for EDS analysis; (e, f) EDS elemental maps of (e) Pt and (f) C.

the RGO is greater than a threshold value for bond breaking, the carbon atoms will be displaced on a sub-picosecond time scale, generating vacancies at their prior sites [45]. In the SAED pattern in Fig. 5d, rings corresponding to the (111), (200), (220), and (420) planes of polycrystalline Pt and the (101) and (112) planes of polycrystalline carbon can be clearly seen . In EDS elemental maps of the TEM image in Figs. 5e-h, some traces of oxygen are observed; this most likely is due to the inclusion of atmospheric oxygen, adsorbed at the many defect sites created by the e-beam irradiation, as well as the oxygen functional groups of the RGO.

Raman spectra of the pristine and Pt-functionalized RGOs showed fingerprints of various carbon allotropes, mostly in the $\mathrm{D}$ and $\mathrm{G}$ peak regions around 1350 and $1580 \mathrm{~cm}^{-1}$, respectively, caused by changes in the electron bands. Identification of these features allows the graphene to be characterized in several ways, including the number of layers present, the effects of strain, doping concentration and
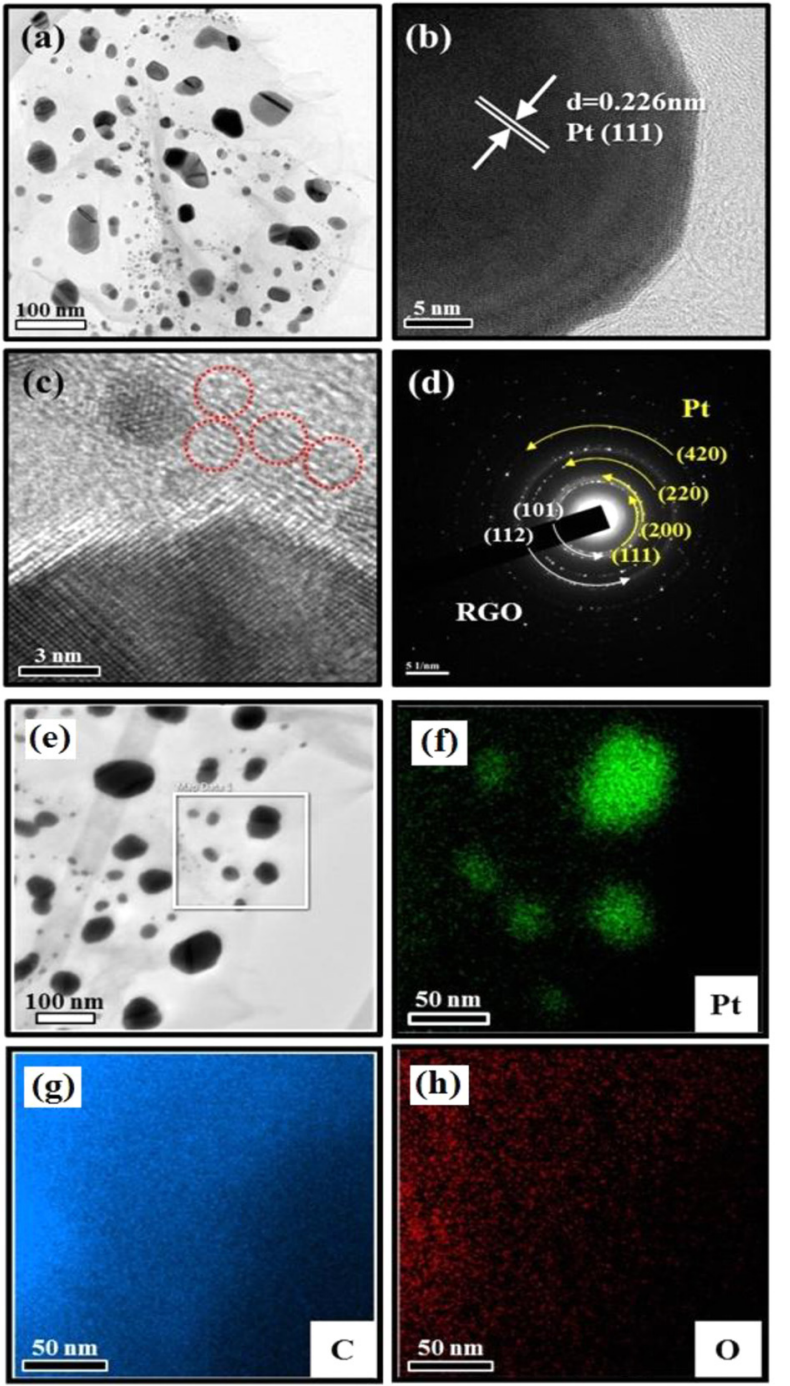

Fig. 5. (a) TEM image of Pt-functionalized RGO irradiated at the dose of $500 \mathrm{kGy}$. (b) High-magnification TEM image. (c) Lattice-resolved TEM image, indicating the presence of defects; (d) corresponding SAED pattern. (e) TEM image for EDS analysis; (f, g) EDS elemental maps of (f) Pt and (g) C.

temperature, and the presence of defects [46]. The main Raman band in graphene, known as the $\mathrm{G}$ band $\left(\sim 1580 \mathrm{~cm}^{-1}\right)$, corresponds to the $E_{2 g}$ mode. The line shape of the $G$ band varies based on the sample quality, but this band exists for all $\mathrm{sp}^{2}$ carbon systems, including amorphous carbon, carbon nanotubes, and graphite [47]. The D band, which is disorder-induced and occurs at around $1350 \mathrm{~cm}^{-1}$ is another important band. Unlike the $\mathrm{G}$ band, to be present the $\mathrm{D}$ band requires defects for momentum conservation [47]. The empirical Tuinstra-Koenig relation [48], which is the ratio of 


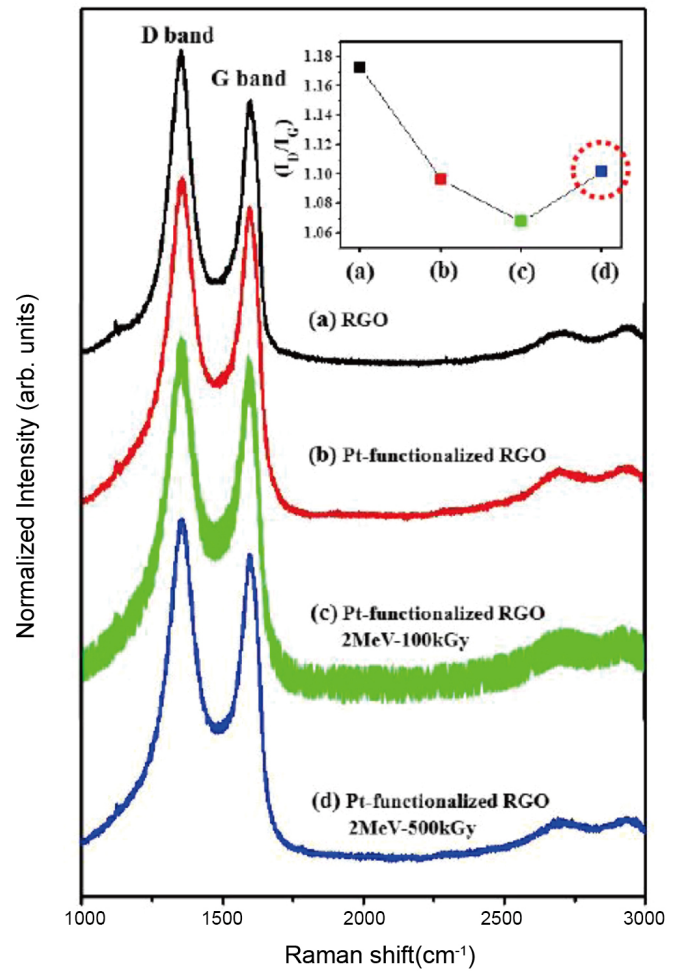

Fig. 6. Raman spectra of (a) pristine RGO, (b) unirradiated Pt-functionalized RGO, (c) Pt-functionalized RGO irradiated at the dose of $100 \mathrm{kGy}$, and (d) Pt-functionalized RGO irradiated at $500 \mathrm{kGy}$.

the intensity of the D and G peaks (ID/IG), represents the degree of order across the graphitic planes and defect levels. Smaller values of this ratio represent greater regularity, and greater ratios indicate more defects and disorder [49]. The ID/IG values of pristine RGO, Pt-functionalized RGO, Pt-functionalized RGO irradiated at the dose of $100 \mathrm{kGy}$, and Pt-functionalized RGO irradiated at $500 \mathrm{kGy}$ were estimated from Raman spectra to be $1.17,1.09,1.07$, and 1.10, respectively. Although providing the exact mechanism for the variations in ID/IG is beyond the scope of this work, we suggest the following qualitative explanation. It is supposed that during the reduction process, many defects are generated in the carbon 2D lattice (including point defects or the incomplete removals of epoxy/oxygenated functional groups) [46]. However, after Pt functionalization, due to the reduction in the RGO surface area, the number of defects is also reduced. Then, during e-beam irradiation, the removal of oxygen functional groups is accompanied by the creation of more defects. However at the same time, the RGO is

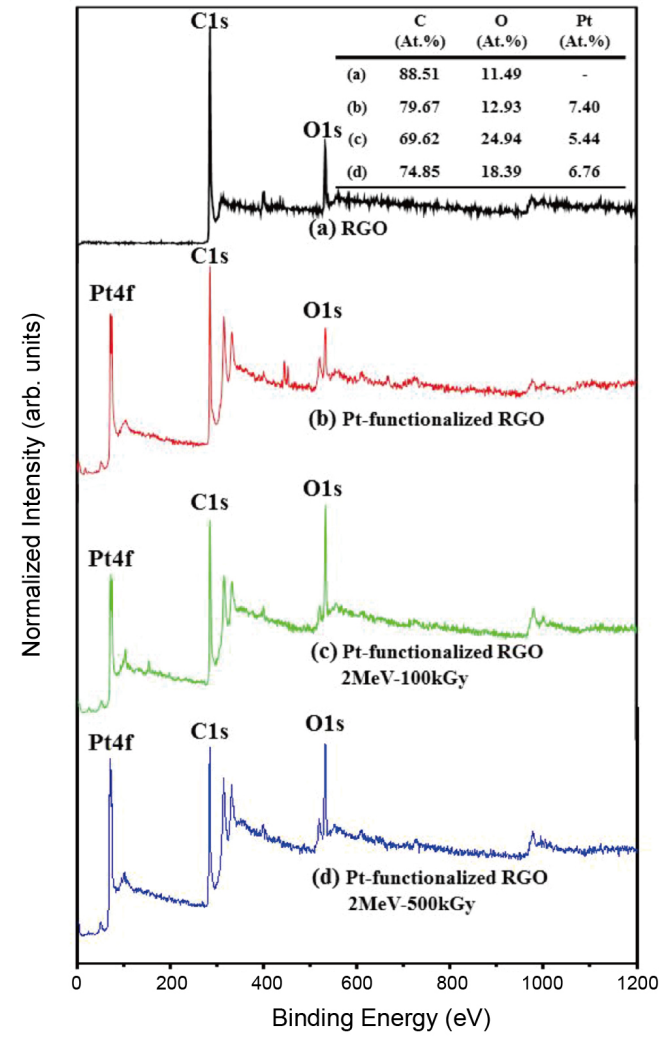

Fig. 7. XPS survey spectra of (a) pristine RGO, (b) unirradiated Pt-functionalized RGO, (c) Pt-functionalized RGO irradiated at the dose of $100 \mathrm{kGy}$, and (d) Pt-functionalized RGO irradiated at $500 \mathrm{kGy}$.

oxidized, which decreases the numbers of defects. In other words, defects were created after irradiation at the $100 \mathrm{kGy}$ dose due to the creation of oxygen vacancies, but oxygen species were also adsorbed from ambient air onto the active defects of RGO, thereby decreasing the number of active defects. After irradiation at the dose of $500 \mathrm{kGy}$, however, there were many more defects, so even after oxidation some active defects remain [50].

Surface chemical analysis of all the film samples was carried out using XPS. In the XPS survey spectra, peaks were observed at around 71.1, 285.5, and 534.0 eV (Fig. 7); these can be assigned to the $\mathrm{Pt}_{4 f}, \mathrm{C}_{1 \mathrm{~s}}$, and $\mathrm{O}_{1 \mathrm{~s}}$ core levels, respectively [51]. In the pristine RGO the atomic percentages of $\mathrm{C}$ and $\mathrm{O}$ were $88.51 \%$ and $11.49 \%$, respectively, and in the unirradiated Pt- functionalized RGO the atomic percentages of $\mathrm{C}, \mathrm{O}$, and $\mathrm{Pt}$ were $79.67 \%, 12.93 \%$, and $7.40 \%$, respectively. It is obvious that the introduction of Pt reduced 

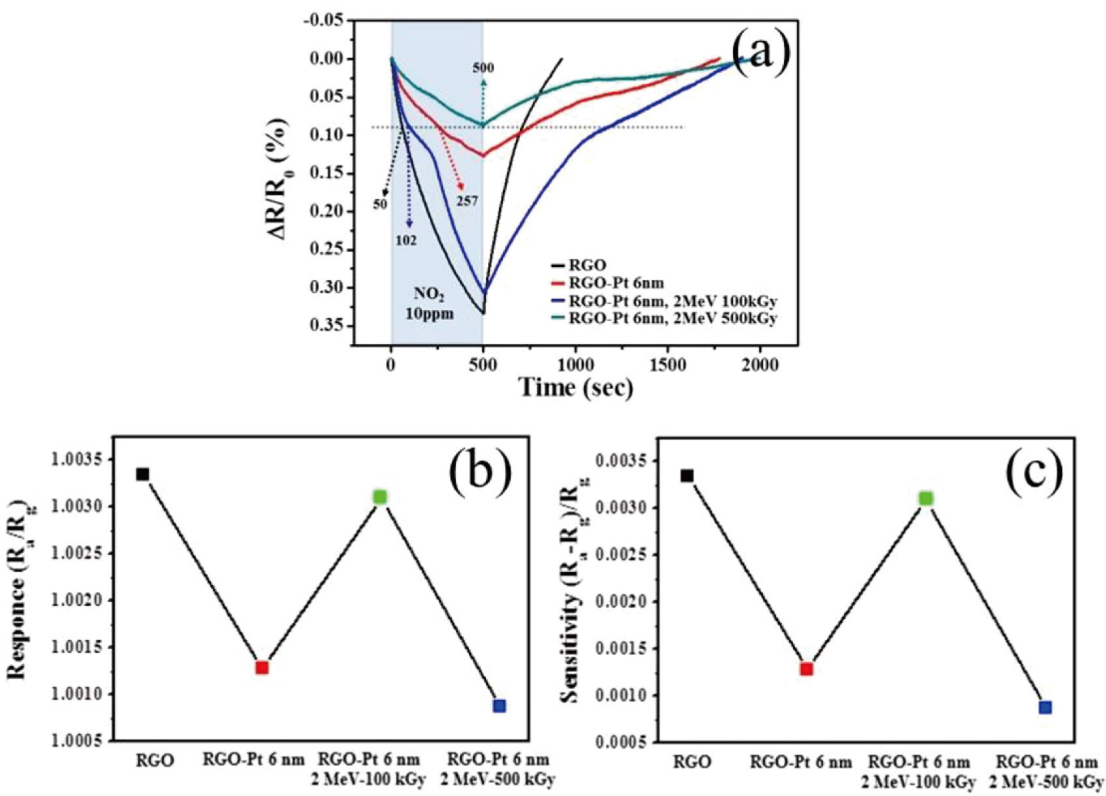

Fig. 8. (a) Dynamic resistance curves, (b) sensor response, and (c) sensitivity of pristine RGO, unirradiated Pt-functionalized RGO, Pt-functionalized RGO irradiated at the dose of $100 \mathrm{kGy}$, and Pt-functionalized RGO irradiated at $500 \mathrm{kGy}$.

the atomic percentage of carbon. E-beam irradiation at the dose of $100 \mathrm{kGy}$ changed the atomic percentages of $\mathrm{C}$, O, and Pt to $69.62 \%, 24.92 \%$, and $5.44 \%$, respectively, and for the sample dosed at $500 \mathrm{kGy}$, the atomic percentages of $\mathrm{C}, \mathrm{O}$, and Pt were $74.85 \%, 18.39 \%$, and $6.76 \%$, respectively. The results indicate that the e-beam irradiation at the dose of 100 kGy created some defects which had a strong tendency to adsorb atmospheric oxygen, so the surface oxygen in this sample was increased. In contrast, less oxygen was present in the sample irradiated with the higher dose, which can be attributed to the fact that substantial amounts of functional oxygen as well as carbon atoms were removed from the surface of the sample.

\subsection{GAS SENSING STUDIES}

Nitrogen dioxide $\left(\mathrm{NO}_{2}\right)$ is one of the toxic gases generated during combustion in factories, cars, and thermal power plants [52]. Sufficiently high amounts of $\mathrm{NO}_{2}$ gas in the environment can cause acid rain and photochemical smog. Human exposure to low concentrations of $\mathrm{NO}_{2}$ can cause irritation to the throat and respiratory system, while higher concentrations can cause more severe respiratory diseases, loss of lung function, and even death [53]. Therefore, for the protection of the environment and human health, there is a growing need for $\mathrm{NO}_{2}$ gas sensors that function at low temperatures and have low power requirements. Accordingly, we studied the room-temperature $\mathrm{NO}_{2}$ gas sensing properties of the fabricated sensors. The transient resistance curves of all sensors towards $10 \mathrm{ppm} \mathrm{NO}_{2}$ gas are shown in Fig. 8a. All of the sensors exhibited p-type behavior: after the introduction of $\mathrm{NO}_{2}$ gas, the resistances were decreased. This means that the irradiation treatment did not change the conducting behavior of the RGO, as the unirradiated sample showed the same p-type sensing behavior. Sensor responses (Fig. 8b) and the sensitivities (Fig. 8c) of the variously prepared sensors were calculated. The pristine RGO, unirradiated Pt-functionalized RGO, Pt-functionalized RGO irradiated at the dose of $100 \mathrm{kGy}$, and Pt-functionalized RGO irradiated at 500 kGy exhibited sensitivities of 0.00335, 0.00129, 0.00310 , and 0.00088 , respectively. It can be observed that the sensitivity of pristine RGO was higher than that of Pt-functionalized RGO dosed at $100 \mathrm{kGy}$, and the sensitivity of unirradiated Pt-functionalized RGO was higher than that of the Pt-functionalized RGO dosed at $500 \mathrm{kGy}$. Therefore, we can conclude that there is some optimum dose of e-beam irradiation for enhancing gas sensing, and that the $100 \mathrm{kGy}$ 
dose was better than the $500 \mathrm{kGy}$ dose in terms of enhancing the response to $\mathrm{NO}_{2}$.

At room temperature, oxygen molecules are adsorbed onto the surface of a sensor and capture electrons from the sensing layer, leaving oxygen adsorbents $\left(\mathrm{O}_{2}{ }^{-}\right)[54]$ :

$\mathrm{O}_{2}$ (gas) $+\mathrm{e}^{-} \rightarrow \mathrm{O}_{2}^{-}$(ads). In an atmosphere containing $\mathrm{NO}_{2}$, the following reactions are expected [55]:

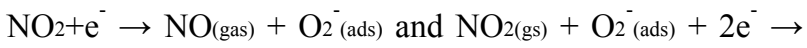
$\mathrm{NO}($ gas $)+\mathrm{O}_{2}^{-}{ }_{(\text {ads })}+2 \mathrm{O}^{-}(\mathrm{ads})$

Thus the resistance of p-typed RGO will be decreased. In the presence of Pt NPs, $\mathrm{NO}_{2}$ gas molecules easily adsorb onto the Pt surfaces and simply transfer to the RGO, where they dissociate into more reactive species [56]. At the interface between the Pt and RGO, the recombination of electron-hole pairs takes place, consequently decreasing the hole concentration in the RGO part [57,58]. Adsorbed $\mathrm{NO}_{2}$ will increase the hole concentrations in the RGO, leading to decreased resistance and increased response compared to that of pristine RGO $[59,60]$. In contrast to the expectation that $\mathrm{Pt}$ functionalization would increase the $\mathrm{NO}_{2}$ sensitivity of the pristine $\mathrm{RGO}$, Pt functionalization decreased $\mathrm{NO}_{2}$ sensitivity from 0.00335 to 0.00129 . Pt functionalization thus appears to have negative impacts which outweighed the expected positive effects of increased sensitivity. According to the XPS results shown in Fig. 7, Pt functionalization increased the oxygen content from 11.49 to $12.93 \%$. This increase may be related to the sputtering process, as it activates ambient oxygen, contributing to the increase in oxygen groups on the RGO surface and to partial oxidation of the RGO. These oxygen groups will reduce the number of defective sites on the RGO surface, which are very important for gas adsorption. For example, An et al. studied adsorption of $\mathrm{NO}_{2}$ onto $\mathrm{ZnO}$ nanotubes [61], finding that $\mathrm{NO}_{2}$ bound almost three times more strongly to defect sites than to the surface of defect-free $\mathrm{ZnO}$ nanotubes $(-0.98 \mathrm{eV}$ versus $-0.30 \mathrm{eV})$. Therefore, defective structures had stronger responses to $\mathrm{NO}_{2}$ gas. Also, during thermal annealing, many surface defects will be deactivated by the presence of Pt NPs. Therefore the decrease in sensitivity to $\mathrm{NO}_{2}$ gas after Pt functionalization can be attributed to the reduction in the native defects of the RGO owing to the sputtering process, thermal annealing, and the existence of Pt NPs.

E-beam irradiation can provide the energy needed to desorb oxygen functional groups from the RGO surface, and after irradiation by e-beams, significant changes in sensitivity were observed. In fact, when energetic electrons strike the RGO surface, if they have sufficient kinetic energy, they can generate surface defects. Oxygen vacancies are the defects most likely to form on the surface of the irradiated RGO, resulting from the detachment of ions from their lattice positions by the high energy of the e-beam irradiation. In addition, e-beams can collide with ambient oxygen molecules and thus generate more reactive and high-energy oxygen species. These oxygen species readily react with the RGO surface to generate oxygen functional groups [62]. Thus, it can be concluded that changes in the sensing characteristics following e-beam irradiation can be attributed to alteration of structural defects and oxygen functional groups. After e-beam irradiation at the dose of $100 \mathrm{kGy}$, the $\mathrm{NO}_{2}$ sensitivity of Pt-functionalized RGO was increased from 0.00129 to 0.00310. On the other hand, the dose of $500 \mathrm{kGy}$ decreased the $\mathrm{NO}_{2}$ sensitivity from 0.00310 to 0.00088 . It can be seen that the $100 \mathrm{kGy}$ irradiation dose was more favorable. XPS results showed that the oxygen content was significantly increased after irradiation with the $100 \mathrm{kGy}$ dose. It can be supposed that the $100 \mathrm{kGy}$ dose created defect sites in the samples which had strong potential to adsorb ambient oxygen. Accordingly, more oxygen could be adsorbed from the atmosphere, partially oxidizing the RGO. $\mathrm{NO}_{2}$ gas molecules can react with these adsorbed oxygens, leading to the greater response.

XRD and XPS analyses revealed that after e-beam irradiation at the $500 \mathrm{kGy}$ dose, the d-spacing and oxygen content of the RGO were decreased relative to the sample dosed at $100 \mathrm{kGy}$. As shown in the TEM image in Fig. 5c (see red circles), the $500 \mathrm{kGy}$ dose generated many structural defects. The reduction in sensitivity observed after irradiation at $500 \mathrm{kGy}$ can be attributed to the generation of many high-energy structural defects on the RGO surface, as well as a possible rupture of the RGO in some locations, which limits 
electrical connections within the sensor. The $500 \mathrm{kGy}$ dose may also generate some non-oxygen defects such as 5-8-5 defects [63] and carbon vacancies, which are detrimental to the $\mathrm{NO}_{2}$ sensitivity. Defects in general act as scattering sites and inhibit charge transport by limiting the electron mean free path when they are connected together, which can be severely detrimental to sensing applications [46]. Such connections may have occurred in the sample dosed at $500 \mathrm{kGy}$. Furthermore, with irradiation at the $500 \mathrm{kGy}$ dose, it is possible that some of the Pt NPs would agglomerate, and thus decrease the sensitizing effect of the Pt [60].

The response times of the pristine RGO, unirradiated Pt-functionalized RGO, Pt-functionalized RGO irradiated at the dose of $100 \mathrm{kGy}$, and Pt-functionalized RGO irradiated at $500 \mathrm{kGy}$ were 52, 257, 102, and $500 \mathrm{~s}$ respectively, and the recovery times for these sensors were 332, 1136, 1166, and $1320 \mathrm{~s}$, respectively. In general, the response and recovery times for the pristine RGO sensor were shorter than those of the Pt-functionalized sensors, with or without e-beam irradiation treatment. This can be attributed to the excellent electrical properties of RGO, which facilitate the transport of charge carriers. The longer response time of the Pt-functionalized RGO sensors can be attributed to the more tortuous structure of the Pt-functionalized samples. The gas diffusion rate is directly proportional to porosity and pore diameter, but is inversely proportional to the tortuosity of the sensor microstructure [64]. Complex channels and pores can be created by e-beams, and this can result in longer times for $\mathrm{NO}_{2}$ molecules to completely adsorb or desorb from the sensor surfaces. The actual the ratios $(\tau)$ of recovery time to response time for pristine RGO, unirradiated Pt-functionalized RGO, Pt-functionalized RGO irradiated at the dose of $100 \mathrm{kGy}$, and Pt-functionalized RGO irradiated at $500 \mathrm{kGy}$ were 6.4, 4.4, 11.4, and 2.6, respectively. Higher values of $\tau$ represent slower desorption relative to adsorption. Usually, gas molecules quickly adsorb on the surface, whereas the desorption process is slow because the bonds between the gas molecules and the sensing material are quite strong. In other words, the adsorption kinetics is faster than the desorption kinetics. The recovery process involves the desorption of $\mathrm{NO}_{2}$, the diffusion of oxygen to the sensing surface, and the adsorption of ambient oxygen gas. The greater $\tau$ of the Pt-functionalized RGO irradiated at the dose of 100 $\mathrm{kGy}$ is likely related to the formation of defect, the degree of complexity of the surface microstructure, the presence of some functional groups on the surface, and so on. Further systematic investigation is needed to clarify the specific mechanism. At this moment, it is not clear why the addition of a Pt layer tends to decrease the sensing behavior of RGO. Since there is a possibility that a Pt layer of $6 \mathrm{~nm}$ is not an optimized condition, we are planning to determine the optimal thickness of the $\mathrm{Pt}$ layer in terms of gas sensitivity, by varying the thickness. The exploration of novel nanomaterials will pave the way to a variety of applications [65-76].

\section{CONCLUSIONS}

Pristine and Pt-functionalized RGO gas sensors were successfully synthesized by reducing GO prepared by means of Hummers' method, followed by the sputtering of Pt layers and thermal annealing. Then the samples were post-treated by irradiation from a 2-MeV electron accelerator at doses of 100 or $500 \mathrm{kGy}$. The structural, chemical, morphological, and $\mathrm{NO}_{2}$ gas sensing properties of the synthesized samples were studied in detail using different techniques. TEM and EDS analyses showed the presence of dispersed Pt NPs on the surfaces of the RGO layers. $\mathrm{NO}_{2}$ gas sensing measurements at room temperature showed that after e-beam irradiation at a dose of $100 \mathrm{kGy}$, the response was increased relative to that of an unirradiated Pt-functionalized sample. This improved performance was attributed to the presence of an optimum amount of defects and functional oxygen groups in the sample. In contrast, when samples were irradiated with a dose of $500 \mathrm{kGy}$, the response was decreased, possibly owing to the formation of a large network of connected defect sites. These results demonstrated the beneficial effect of e-beam irradiation for improving the gas sensing response of Pt-functionalized RGO.

\section{ACKNOWLEDGEMENTS}

This research was supported by Basic Science Research Program through the National Research Foundation of Korea 
(NRF) funded by the Ministry of Education (2016R1A6A1A03013422). This work was supported by the National Research Foundation of Korea (NRF) Grant funded by the Korean Government (MSIP) (No. Grant Number 2015R1A5A7037615).

\section{REFERENCES}

1. K. Bhowmik and A. Mondal, Electron. Mater. Lett. 11, 180 (2015).

2. D. N. Andoshe, J. -M. Jeon, S. Y. Kim, and H. W. Jang, Electron. Mater. Lett. 11, 180 (2015).

3. S. P. Khatkar, S. Singh, S. Lohra, A. Khatkar, and V. Taxak, Electron. Mater. Lett. 11, 409 (2015).

4. T. -J. Ha and A. Lee, Electron. Mater. Lett. 11, 552 (2015).

5. M. O. Ansari, M. M. Khan, S. A. Ansari, and M. H. Cho, Electron. Mater. Lett. 11, 559 (2015).

6. G. Anoop, E. Y. Park, S. Lee, and G. Y. Jo, Electron. Mater. Lett. 11, 565 (2015).

7. S. Park, G. -J. Sun, S. Kim, S. Lee, and C. Lee, Electron. Mater. Lett. 11, 572 (2015).

8. P. Kondaiah, H. Shaik, and G. M. Rao, Electron. Mater. Lett. 11, 592 (2015).

9. M. Karimipour, S. Mashhoun, M. Mollaei, M. Molaei, and N. Taghavinia, Electron. Mater. Lett. 11, 625 (2015).

10. D. Jung, S. G. Cho, T. Moon, and H. Sohn, Electron. Mater. Lett. 12, 17 (2016).

11. X. Lian, Y. Li, T. Lv, Y. Zou, D. An, and N. Zhang, Electron. Mater. Lett. 12, 17 (2016).

12. M. Karimipour and M. Molaei, Electron. Mater. Lett. 12, 205 (2016).

13. M. J. Kiani, E. Akbari, F. R. Kooshkaki, and A. Zeinalinezhad, Electron. Mater. Lett. 12, 219 (2016).

14. T. U. Kim, M. G. Gang, J. A. Kim, J. H. Moon, D. G. Kim, S. H. Kim, H. C. Ki, J. H. Choi, and J. H. Kim, Electron. Mater. Lett. 12, 224 (2016).

15. W. J. Meang, J. Seo, Y. Ahn, and J. Y. Son, Electron. Mater. Lett. 12, 251 (2016).

16. N. T. N. Truong and C. Park, Electron. Mater. Lett. 12, 308 (2016).

17. V. Kannan, H. S. Kim, and H. C. Park, Electron. Mater. Lett. 12, 323 (2016).

18. Z. Chen, Z. Lin, M. Xu, Y. Hong, N. Li, P. Fu, and Z. Chen, Electron. Mater. Lett. 12, 343 (2016).

19. H. Kim, S. J. Kim, K. Y. Ko, H. Kim, J. Kim, J. Oh, and H.B.R. Lee, Electron. Mater. Lett. 12, 404 (2016).

20. Y. Li, T. Lv, F.-X. Zhao, X.-X. Lian, Y.-L. Zou, and Q. Wang, Electron. Mater. Lett. 12, 411 (2016).

21. J. T. Robinson, F. K. Perkins, E. S. Snow, Z. Q. Wei, and P. E. Sheehan, Nano Lett. 8, 3137 (2008).

22. S. Deng, V. Tjoa, H. M. Fan, H. R. Tan, D. C. Sayle, M. Olivo, S. Mhaisalkar, J. Wei, and C. H. Sow, J. Am.
Ceram. Soc. 134, 4905 (2012).

23. S. Bai, C. Chen, R. Luo, A. Chen, and D. Li, Sens. Actuators B Chem. 216, 113 (2015).

24. J. H. Kim, P. Wu, H. W. Kim, and S. S. Kim, ACS Appl. Mater. Interfaces 8, 7173 (2016).

25. I. Childres, L. A. Jauregui, M. Foxe, J. Tian, R. Jalilian, I. Jovanovic, and Y. P. Chen, Appl. Phys. Lett. 97, 173109 (2010).

26. P. Tyagi, S. Sharma, M. Tomar, F. Singh, and V. Gupta, Nuc. Instrum. Methods Phys. Res. B 379, 219 (2016).

27. K. Yin, S. Liu, Q. Cai, A. Gao, S. Lu, and M. Shao, J. Mater. Sci.: Mater. Electron. 25, 419 (2014).

28. N. A. Sabu, K. P. Priyanka, S. Ganesh, and T. Varghese, Radiat. Phys. Chem. 123, 1 (2016).

29. V. V. Emtsev, V. Y. Davydov, V. V. Kozlovskii, V. V. Lundin, D. S. Poloskin, A. N. Smirnov, N. M. Shmidt, A. S. Usikov, J. Aderhold, H. Klausing, and D. Mistele, Semicond. Sci. Technol. 15, 73 (2000).

30. K. Yin, S. Liu, Q. Cai, A. Gao, S. Lu, and M. Shao, J. Mater. Sci.: Mater. Electron. 25, 419 (2014).

31. A. K. Bal, R. Singh, and R. K. Bedi, J. Mater. Sci. Technol. 28, 700 (2012).

32. Y. J. Kwon, H. Y. Cho, H. G. Na, B. C. Lee, S. S. Kim, and H. W. Kim, Sens. Actuators B Chem. 203, 143 (2014)

33. J. Gong, H. Liu, Y. Jiang, S. Yang, X. Liao, Z. Liu, and S. Ringer, Mater. Charact. 100, 1 (2015).

34. J. Kugai, T. Moriya, S. Seino, T. Nakagawa, Y. Ohkubo, H. Nitani, and T. A. Yamamoto, Int. J. Hydrogen Energy 38, 4456 (2013).

35. L. A. P. Corrales, A. L. Claveríe, and K. F. Arndt, React. Funct. Polym. 75, 31 (2014).

36. B. Christopher, A. Rao, V. C. Petwal, V. P. Verma, J. Dwivedi, W. J. Lin, and Y. K. Kuo, Physica B: Condens. Matter. 502, 119 (2016).

37. N. Lavanya, C. Sekar, A. C. Anithaa, N. Sudhan, K. Asokan, A. Bonavita, S. G. Leonardi, and G. Neri, Nanotechnology 27, 385502 (2016).

38. H. Yu, P. Xu, D. W. Lee, and X. Li, J. Mater. Chem. A 1, 4444 (2013).

39. F. T. Johra and W. G. Jung, Appl. Surf. Sci. 362, 169 (2016).

40. A. Esfandiar, S. Ghasemi, A. Irajizad, O. Akhavan, and M. R. Gholami, Int. J. Hydrogen Energy 37, 15423 (2012).

41. W. S. Hummers and R. E. Offeman, J. Am.Chem. Soc. 80, 1339 (1958).

42. Y. J. Kwon, H. Y. Cho, H. G. Na, B. C. Lee, S. S. Kim, and H. W. Kim, Sens. Actuators B Chem. 203, 143 (2014).

43. Y. J. Kwon, H. G. Na, S. Y. Kang, M. S. Choi, J. H. Bang, T. W. Kim, A. Mirzaei, and H. W. Kim, Sens. Actuators B Chem. 239, 180 (2017).

44. K. K. Babitha, K. P. Priyanka, A. Sreedevi, S. Ganesh, and T. Varghese, Mater. Charact. 98, 222 (2014).

45. H. Terrones, R. Lv, M. Terrones, and M. S. Dresselhaus, 
Rep. Prog. Phys. 75, 062501 (2012).

46. V. Singh, D. Joung, L. Zhai, S. Das, S. I. Khondakera, and S. Seal, Prog. Mater. Sci. 56, 1178 (2011).

47. R. Beams, L. G. Cancado, and L. Novotny, J. Phys.: Condens. Matter. 27, 083002 (2015).

48. F. Tuinstra and Koenig, J. Chem. Phys. 53, 1126 (1970).

49. A. Esfandiar, A. Irajizad, O. Akhavan, S. Ghasemi, and M. R. Gholami, Int. J. Hydrogen Energy 39, 8169 (2014).

50. D. Chen, J. Jiang, and X. Du, Talanta 155, 329 (2016).

51. M. Brun, A. Berthet, and J. C. Bertolini, J. Electron Spectros. Relat. Phenomena 104, 55 (1999).

52. S. S. Shendage, V. L. Patil, S. A. Vanalakar, S. P. Patil, N. S. Harale, J. L. Bhosale, J. H. Kim, and P. S. Patil, Sens. Actuators B Chem. 240, 426 (2017).

53. K. Wetchakun, T. Samerjai, N. Tamaekong, C. Liewhiran, C. Siriwonga, V. Kruefu, A. Wisitsoraat, A. Tuantranont, and S. Phanichphant, Sens. Actuators B Chem. 160, 580 (2011).

54. S. W. Choi, S. H. Jung, and S. S. Kim, J. Hazard. Mater. 193, 243 (2011).

55. U. Yaqoob, D. T. Phan, A. S. M. I. Uddin, and G. S. Chung, Sens. Actuators B Chem. 211, 760 (2015).

56. H. Kim, S. Park, C. Jin, and C. Lee, Nano Energy 6, 455 (2011).

57. A. Mirzaei, K. Janghorban, B. Hashemi, and G. Neri, J. Nanopart. Res. 17, 1 (2015).

58. Z. U. Abideen, A. Katoch, J. H. Kim, Y. J. Kwon, H. W. Kim, and S. S. Kim, Sens. Actuators B Chem. 211, 1499 (2015).

59. J. Wang, S. Rathi, B. Singh, I. Lee, S. Maeng, H. I. Johd, and G. H. Kim, Sens. Actuators B Chem. 220, 755 (2015).

60. R. Ghosh, S. Santra, S. K. Ray, and P. K. Guha, Appl.
Phys. Lett. 107, 153102 (2015).

61. W. An, X. J. Wu, and X. C. Zeng, J. Phys. Chem. C. 112, 5747 (2008).

62. M. G. Chung, D. H. Kim, H. M. Lee, T. Kim, J. H. Choi, D. K. Seo, J. B. Yoo, S. H. Hong, T. J. Kang, and Y. H. Kim, Sens. Actuators B Chem. 166-167, 172 (2013).

63. A. Hashimoto, K. Suenaga, A. Gloter, K. Urita, and S. Iijima, Nature 430, 870 (2004).

64. J. Huang, Y. Wu, C. Gu, M. Zhai, K. Yu, M. Yang, and J. Liu, Sens. Actuators B Chem. 206, 212 (2010).

65. S. Park, G.-J. Sun, H. Kheel, S. K. Hyun, C. Jin, and C. Lee, Met. Mater. Int. 22, 156 (2016).

66. J.-H. Choi, J. Kim, S. J. Oh, D. Kim, Y.-H. Kim, H. Chae, and H. Kim, Met. Mater. Int. 22, 723 (2016).

67. B. W. Lee, D. H. Cho, J. H. Nam, and I. M. Park, Met. Mater. Int. 22, 714 (2016).

68. J. A. Desai and A. Kumar, Met. Mater. Int. 22, 451 (2016).

69. H. Puga, V. H. Carneiro, J. Barbosa, and D. Soares, Met. Mater. Int. 22, 863 (2016).

70. E. Danaila, L. Benea, N. Caron, and O. Raquet, Met. Mater. Int. 22, 924 (2016).

71. H.-S. Yun, J.-C. Kim, D.-Y. Jeong, and N. H. Cho, Met. Mater. Int. 22, 1108 (2016).

72. S.-H. Hong and M. Y. Song, Met. Mater. Int. 22, 1121 (2016).

73. F. Khan, S.-H. Baek, N. Ahmad, G. H. Lee, T. H. Seo, E.-K. Suh, and J. H. Kim, Met. Mater. Int. 21, 561 (2015).

74. G. F. Li, H. Z. Zheng, X. Y. Shu, and P. Peng, Met. Mater. Int. 22, 69 (2016).

75. I.-J. Shon, Korean J. Met. Mater. 54, 826 (2016).

76. I.-J. Shon, Korean J. Met. Mater. 54, 893 (2016). 